\title{
Detecting deception in a bluffing body: The role of expertise
}

\author{
Natalie Sebanz \\ Radboud University Nijmegen, Nijmegen, The Netherlands \\ AND \\ MAGGIE SHIFFRAR \\ Rutgers University, Newark, New Jersey
}

\begin{abstract}
Studies of deception detection traditionally have focused on verbal communication. Nevertheless, people commonly deceive others through nonverbal cues. Previous research has shown that intentions can be inferred from the ways in which people move their bodies. Furthermore, motor expertise within a given domain has been shown to increase visual sensitivity to other people's movements within that domain. Does expertise also enhance deception detection from bodily movement? In two psychophysical studies, experienced basketball players and novices attempted to distinguish deceptive intentions (fake passes) and veridical intentions (true passes) from an observed individual's actions. Whereas experts and novices performed similarly with postural cues, only experts could detect deception from kinematics alone. These results demonstrate a link between action expertise and the detection of nonverbal deception.
\end{abstract}

Successful social interaction requires the rapid detection of other people's identities and intentions (Frith \& Frith, 2006). A rich source of information lies in the ways that people move their bodies. Research over the last three decades indicates that the visual analysis of bodily movement is sufficient to determine other people's identities (e.g., Cutting \& Kozlowski, 1977; Loula, Prasad, Harber, \& Shiffrar, 2005), moods (e.g., Chouchourelou, Matsuka, Harber, \& Shiffrar, 2006; Dittrich, Troscianko, Lea, \& Morgan, 1996), personality traits (Heberlein, Adolphs, Tranel, \& Damasio, 2004), expectations (Bosbach, Cole, Prinz, \& Knoblich, 2005; Grèzes, Frith, \& Passingham, 2004b), and deceptive intentions (Grèzes, Frith, \& Passingham, 2004a; Runeson \& Frykholm, 1983).

Understanding how deceptive intents can be detected from bodily actions has important consequences for both real-world issues and theories of mental state inference. In the classic paradigm of Runeson and Frykholm (1983), participants watch an actor lift boxes of different weights and judge whether the actor is trying to deceive them about the box's weight. Although far from perfect, naive observers are above chance in their ability to identify deceptive actions directed toward objects (Grèzes et al., 2004a; Runeson \& Frykholm, 1983).

Studies on the impact of nonverbal cues during verbal communication (e.g., Ekman \& Friesen, 1969; Vrij, 2006) have shown that both experts and novices rely heavily on nonverbal cues when judging a person's veracity (Vrij, 2004). Experts tend to attend to the wrong cues and, as a result, their judgments are surprisingly poor (Vrij, 2004). Such research suggests that experts might be no better, or even worse, than novices at detecting deception from movement. However, these studies have not investigated situations in which the body is consciously used as an instrument for deception. Rather, the focus has been on nonverbal signals that leak out without the individual's awareness (Vrij, 2006). Such a passive perspective on the body does not capture situations wherein movements are designed to be deceptive, such as when people fake injuries.

Numerous studies from sports psychology have provided evidence that experts are better than novices at predicting the outcome of observed actions (e.g., Abernethy, 1989; Abernethy \& Zawi, 2007; Müller, Abernethy, \& Farrow, 2006; for a review, see Jackson, Warren, \& Abernethy, 2006), such as predicting the landing position of a volleyball or tennis serve. However, to our knowledge, only one published study has investigated whether expertise affects the detection of deceptive movements (Jackson et al., 2006). This study showed that expert rugby players were better than novices at detecting deceptive moves, such as when an attacking player fakes a cut to the right and then cuts to the left. These findings provide the first evidence that visual and/or motor expertise in a particular domain can improve one's ability to read intentions from other people's moving bodies.

The aim of the present study was to extend this evidence in two ways. First, we wanted to determine whether expertise influences deception detection in another domain. The

N.Sebanz, n.sebanz@donders.ru.nl 
deceptive actions studied by Jackson et al. (2006) were performed to mislead others about the direction in which the deceiver was moving. Changes in running direction are large-scale, salient events. We chose to investigate the detection of basketball fakes, because these deceptive actions are more subtle and are likely more difficult to detect. Second, the aim of our study was to investigate what kinds of cues experts draw on to detect deception. The study by Jackson et al. provided important insights into when an actor's intention can be inferred, but further exploration is needed to identify what kind of information is used during deception detection. We conducted two psychophysical experiments to determine how well experienced and novice basketball players can distinguish intentionally deceitful actions (fake passes) from veridical actions (true passes) based on partial observation of the unfolding action. Experiment 1 compared deception detection from static and dynamic cues, whereas Experiment 2 investigated whether dynamic cues alone are sufficient for the detection of deceptive intention.

\section{EXPERIMENT 1}

In Experiment 1, participants viewed videos and static pictures of a basketball player making passes and fakes (see Figure 1A). Videos showed the player dribbling the ball twice while stepping forward and then picking the ball up as if to throw it. The videos stopped just before the player was about to throw the ball (pass) or mimic a throw (fake). This last frame of each movie was used as the static control image, showing the player's posture just before the pass or fake. We compared how well experts and novices could infer the player's intention when dynamic and/or static information was available.
A

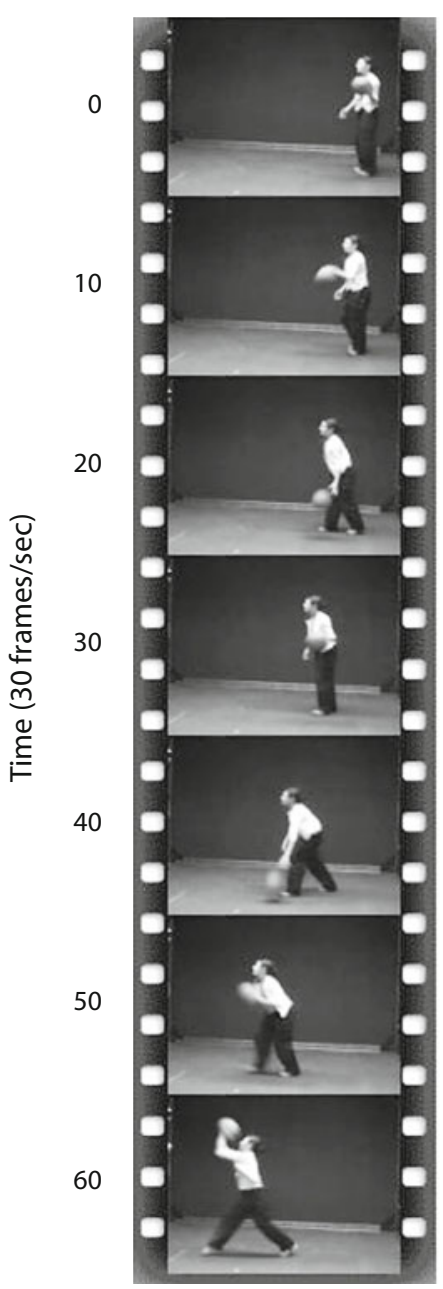

B

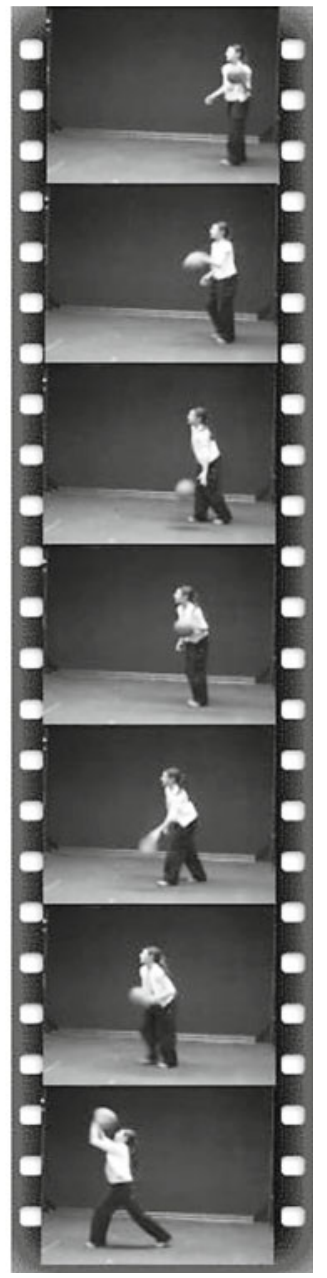

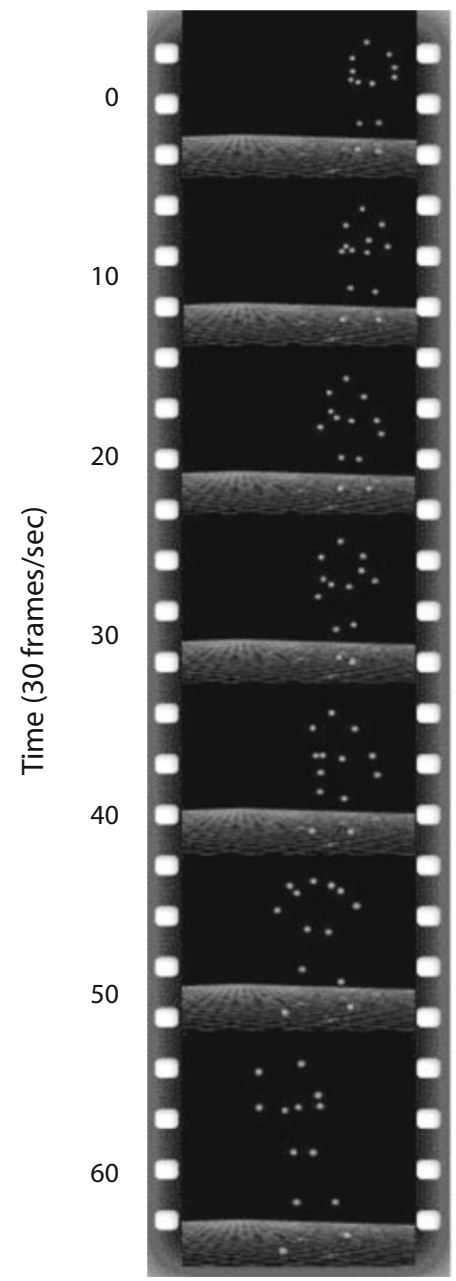

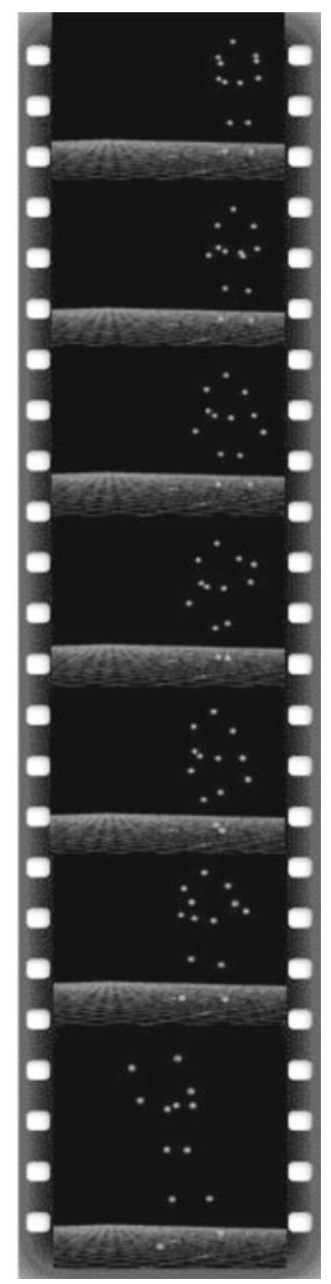

Figure 1. Stimuli used in Experiments 1 and 2. (A) Single frames from the movies in Experiment 1. (B) Single frames from the pointlight movies in Experiment 2; dots represent the locations of the actor's joints. Each column shows seven frames, taken at 10-frame intervals ( $0.33 \mathrm{sec}$ ), from one of the movies displaying a fake (left column) and from one of the movies displaying a pass (right column). The last frame of each movie in panel A corresponds to the static picture derived from this movie. If you are seeing this page on a computer screen, note that you can see the player's movements by scrolling down the page by using the computer mouse. 


\section{Method}

Participants. We recruited 30 participants through advertising at Rutgers University in Newark. They participated for course credit or payment. All had normal or corrected-to-normal vision, were naive as to the purpose of the experiment, and gave informed consent. Twelve participants ( 9 males, 3 females; mean age, 21.3 years) were classified as basketball experts on the basis of a questionnaire they completed prior to the experiment. All had been playing basketball for several years (average, 12.4 years) and regularly played at least once a week. None of the novices (10 males; mean age, 26.0 years) played basketball on a regular basis, either currently or in the past. Experts reported playing $3.4 \mathrm{~h}$ per week on average $(S D=2.3)$ and watching 3 games per week on average $(S D=1.74)$, whereas novices reported watching an average of 0.7 games per week $(S D=$ 0.83 ).

Stimuli. To create the stimulus material, we filmed a basketball player interacting with two other people: one "teammate," to whom she passed the ball, and one "defender," whom she tried to deceive. The video was edited so that these two people could not be seen. The player was instructed to decide prior to each interaction whether she would fake or make a pass. To ensure that the fakes were convincing, the stimuli were constructed only from video clips in which the defender had been completely deceived by the fake. The resultant stimuli consisted of 30 movies depicting passes and 30 movies depicting fakes. The movies were cut so that they ended one frame before the ball left the player's hands (passes) or just before the player withdrew her hands (fakes). For both passes and fakes, one third of the movies displayed overhead passes, one third displayed chest-level passes, and one third displayed bounce passes. Static picture stimuli were cut from the last frame in each movie. Average movie length was $1.9 \mathrm{sec}(S D=0.1 \mathrm{sec})$, with no significant duration difference between passes and fakes $[t(60)=0.33, p=.74]$. In the analyses reported below, results were averaged across the three different pass/ fake types (overhead, chest-level, bounce), because there were no significant interactions involving pass/fake type.

Procedure. On each trial, participants reported whether the player intended to fake or make a pass. Participants were informed that they would see an equal number of passes and fakes. Presentation of movies and pictures was blocked, and the order of conditions was counterbalanced across participants. The sequence of events on each trial was as follows: A black fixation cross appeared on the screen for $100 \mathrm{msec}$, followed by either a movie or a picture. Pictures and movies subtended $15^{\circ} \times 13^{\circ}$ of visual angle horizontally and vertically. As soon as a movie stopped playing, it disappeared from the screen. Static pictures were displayed for $4 \mathrm{sec}$. Participants had an unlimited response-time window after the pictures disappeared, and pressed a key on the computer keyboard marked "P" for pass or a key marked "F" for fake. As soon as their keypress was recorded, the participants could start the next trial via a mouse click. No feedback was given. At the end of the experiment, participants responded to two open questions in writing, describing what kind of strategies they had relied on when making their judgments for movies and pictures.

\section{Results and Discussion}

Figure 2 shows the percentage of correct responses for experts and novices in this deception detection task. Two-sided $t$ tests comparing performance against chance $(50 \%)$ showed that experts and novices were significantly above chance in both conditions (all $p$ s $<.05$ ). Thus, experts and novices were able to infer the player's intention from movement and static posture. More importantly, however, experts performed the deception detection task significantly better than novices when movement was present $[t(28)=2.1, p<.05]$ but showed no advantage with posture alone $[t(28)=0.65, p=.53]$. Accordingly, a $2 \times 2$ repeated measures ANOVA with expertise as a between-subjects factor and condition (movie vs. picture)

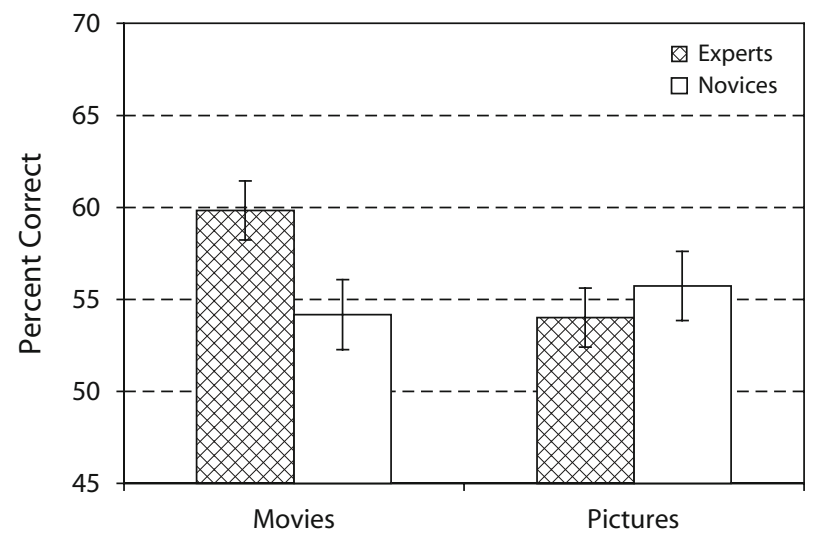

Figure 2. Results of Experiment 1. The bars show the average percentage of correct responses (hits) for passes and fakes. Note that in our task, false alarms to one kind of stimulus are redundant with hits to the other kind of stimulus (responses to fakes can be hits or false alarms to passes, and responses to passes can be hits or false alarms to fakes). Accordingly, the results are not expressed as a measure of $d^{\prime}$, but in percent correct, where $50 \%$ is chance. Expressing accuracy in terms of percent correct is common in research on deception (Bond \& DePaulo, 2006).

as a within-subjects factor showed a significant interaction between expertise and condition $[F(1,28)=9.29$, $\left.p_{\text {rep }}>.99, \eta^{2}=.25\right]$.

These findings suggest two conclusions. First, expertise in a particular action domain improves one's ability to detect deceptive intentions from bodily actions that fall within that same domain. This confirms and extends previous work by Jackson et al. (2006). Second, the fact that experts made more accurate predictions than did novices only when dynamic movement information was available indicates that experts may have tapped into their own action repertoire (Blakemore \& Frith, 2005; Wilson \& Knoblich, 2005), in line with experimental evidence showing that the observation of actions that one knows how to perform activates corresponding representations in the observer's own motor system (Buccino et al., 2004; Calvo-Merino, Glaser, Grèzes, Passingham, \& Haggard, 2005; Calvo-Merino, Grèzes, Glaser, Passingham, \& Haggard, 2006; Cross, Hamilton, \& Grafton, 2006; Rizzolatti \& Craighero, 2004). It is also possible that experts' extensive visual exposure to passes and fakes selectively enhanced their sensitivity to cues expressed in dynamic displays (Jackson et al., 2006).

The questionnaires handed out at the end of the experiment provided evidence suggesting that novices are much more likely than experts to focus on postural form and facial cues. On the basis of written answers, we counted how often participants reported relying on postural, facial, and dynamic cues. Only data from those participants who agreed to fill out the questionnaire and who provided meaningful answers will be reported (9 experts, 12 novices). Postural cues that were mentioned were the position of the feet and the position of the hands; dynamic cues included "energy," "body motion," and "step pattern." Two thirds of the experts did not mention a postural or facial cue, whereas more than $80 \%$ of the novices mentioned at least one postural or facial cue. A chi-square test con- 
firmed that this difference was significant $\left[\chi^{2}(1)=5.45\right.$, $p<.05]$. Although one should certainly not overemphasize these questionnaire results, it is interesting to note that the self-report data fit nicely with the observed differences in perceptual judgments.

\section{EXPERIMENT 2}

Given the results of Experiment 1, a straightforward prediction is that experts should be able to detect another's intentions from kinematic information alone, whereas novices should be at a loss when postural cues, such as foot and hand position, as well as facial expression, are removed. To test this prediction, we created point-light displays of the basketball player. Participants in this experiment saw a small number of moving dots representing the locations of the actor's limb joints (Figure 1B). When set in motion, such stimuli create a vivid impression of human movement (Johansson, 1973) and allow observers to infer extensive information about the actor, including identity (Loula et al., 2005) and emotional state (Chouchourelou et al., 2006). No static pictures were used in this experiment, because observers cannot detect the human form in static point-light displays.

Instead, we investigated whether viewpoint affects deception detection by presenting the same point-light movies with the basketball player approaching the observer $\left(0^{\circ}\right.$, front view) or moving diagonally $\left(30^{\circ}\right.$, half-profile view). Previous studies have shown improved recognition of gender and identity when point-light walkers are viewed from the front, as compared with a half-profile view (Troje, Westhoff, \& Lavrov, 2005). In contrast, studies on face recognition suggest that a half-profile view is the more informative one (Troje \& Bülthoff, 1996). Moreover, basketball players may see others' passes and fakes more often from a side angle than from a front view. By manipulating viewpoint, we hoped to determine whether making judgments about mental states (the other's intention) follows principles similar to those followed when judgments about bodily features are made.

\section{Method}

Participants. Twenty-two new participants were recruited for Experiment 2. Fourteen were experts ( 10 males, 4 females; mean age, 19.5 years), who had been playing basketball for an average of 8.5 years. They reported currently playing $7.5 \mathrm{~h}$ per week $(S D=$ $7.3)$ and watching 2.7 games per week $(S D=1.97)$. The novices ( 3 males, 5 females; mean age, 20.6 years) reported watching 0.5 games per week $(S D=1.41)$.

Stimuli. To create the stimuli for Experiment 2, the same basketball player's movements were recorded with a ReActor motion capture system from Ascension Technology (recording rate, 30 frames/sec). The player wore a specially designed suit to which a set of 30 motion markers was attached. Sensors provided spatiotemporal measures of the actors' limb and head movements. The resulting sensor measurements were subsequently converted to point-light displays showing 13 blue dots, distributed along the major joints and head of the basketball player's body. Motion Builder 5.0 by Kaydara and iMovie software packages were used for movie processing and editing.

From the motion-capture data, we created 12 point-light movies depicting basketball passes and 12 depicting fakes. For passes and fakes, half of the movies showed overhead passes/fakes, and half

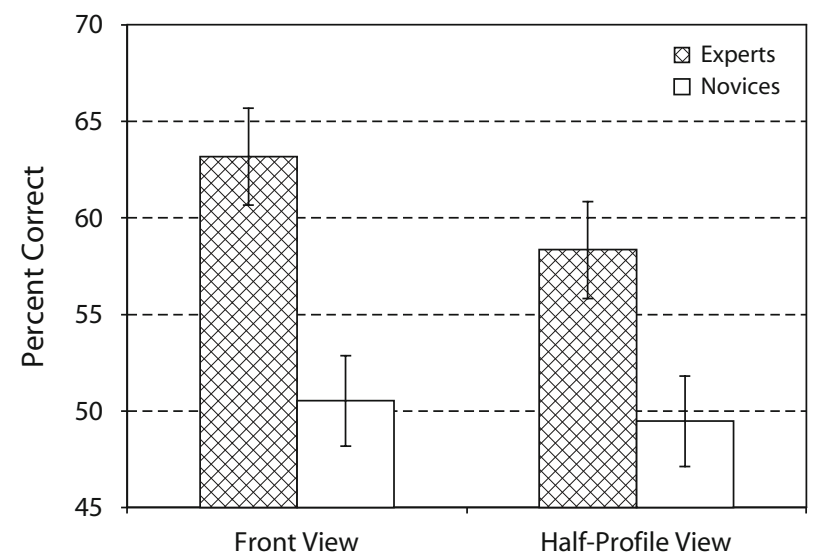

Figure 3. Results of Experiment 2. In both conditions, deception detection performance was at chance $(50 \%)$ for novices but was significantly above chance for basketball experts.

showed bounce passes/fakes. Again, the movies were cut so that the action outcome was ambiguous. Average movie length was $2.5 \mathrm{sec}$ $(S D=0.26 \mathrm{sec})$, with no significant difference between passes and fakes $[t(22)=0.92, p=.37]$. There was a slightly longer duration of the movies in Experiment 2 than in Experiment 1, because pointlight displays take longer to organize perceptually (Johansson, 1973). The point-light figure became slightly larger as it approached the observer (Figure 1B). The two views were created by rotating the point-light figure, so that the actor appeared to face the observer directly $\left(0^{\circ}\right)$ or was seen in half profile $\left(30^{\circ}\right)$. Thus, the same movies were shown from front and half-profile views. In sum, a total of 48 stimuli depicted two types of passes from two viewpoints.

Procedure. The procedure from Experiment 1 was used, with the exception that only movies were presented (front and half-profile views). The order of condition (front vs. half-profile view) was blocked and counterbalanced.

\section{Results and Discussion}

Figure 3 shows the percentage of correct responses. A 2 (experts vs. novices) $\times 2$ (front vs. half-profile view) ANOVA was conducted on percentage of accuracy data. Novices performed at chance regardless of viewpoint. In contrast, experts were able to infer the player's intention from both views [main effect expertise: $F(1,20)=12.58$, $\left.p_{\text {rep }}>.99, \eta^{2}=.14\right]$. This demonstrates that our experts could detect deception from kinematic cues alone. In line with earlier findings on the recognition of gender and identity, experts performed better when they saw the player from the front than when they saw the player from the halfprofile view $[t(13)=2.27, p<.05]$. This suggests that the dynamic features that experts extracted to infer the player's intention were primarily expressed and/or most visible in the frontoparallel plane (Troje et al., 2005). At the same time, this finding provides evidence that the front-view advantage may be much more general than previously assumed, applying to judgments about physical features as well as to judgments about mental states.

\section{GENERAL DISCUSSION}

The idea that deceptive intentions "leak out" through nonverbal bodily cues is well established and extensively 
researched (Vrij, 2006). Much less attention has been paid to how the body is used intentionally as an instrument of deception and how such deception can be detected. Our experiments revealed two main findings. First, observing body movements allows individuals to infer whether an actor intends to deceive others about the course of his or her actions. This extends previous studies that have investigated the detection of object-directed deception (Grèzes et al., 2004a; Runeson \& Frykholm, 1983) and the detection of deceptive rugby moves (Jackson et al., 2006). More importantly, our findings demonstrate that expertise in a particular action domain influences how well deceptive actions, such as a fake pass, can be detected. In line with previous studies on the role of expertise in predicting action outcomes, we found that experts were better than novices at judging whether a perceived action would lead to a pass or a fake.

To our knowledge, this is the first study to directly compare deception detection from dynamic and static cues. Our findings suggest that experts and novices used different strategies when trying to infer the player's intention. Novices appeared to rely on form information, because their performance in Experiment 1 did not improve significantly with the addition of dynamic cues. Indeed, when presented with point-light displays that contained only dynamic cues, novices were unable to infer the actor's intention. In contrast, experts were equally, if not better, able to infer the actor's intention when presented with kinematics alone.

What kind of expertise did the basketball experts draw upon? There are two possibilities that are not mutually exclusive, even though researchers rarely consider both (but see Canal-Bruland \& Schmidt, 2008). On the one hand, basketball players have substantial visual expertise, having watched thousands of passes and fakes. On the other hand, experts also have developed extensive motor expertise from physically performing countless passes and fakes. The present experiments were not designed to rule out either possibility, since both types of expertise contribute to the visual perception of bodily motion (Loula et al., 2005). Although visual expertise plays a significant role in experts' perceptual judgments (Behrmann \& Ewell, 2003; Jacobs, Pinto, \& Shiffrar, 2004), previous research suggests that motor expertise likely has a significantly larger impact (Loula et al., 2005).

Behavioral and brain imaging studies have demonstrated that action perception activates the observer's motor system (Rizzolatti \& Craighero, 2004) and that the degree of similarity between perceived actions and the observer's repertoire of executable actions determines how strongly the motor system is activated (CalvoMerino et al., 2005; Calvo-Merino et al., 2006; Cross et al., 2006) and how accurately action outcomes can be predicted (Knoblich \& Flach, 2001). Thus, basketball experts in the present studies may have relied on simulation processes in their own motor system to infer the observed basketball player's intentions. In particular, the predictive mechanisms used to anticipate the sensorimotor consequences of one's own actions may be employed to predict the future outcomes of others' actions (Blakemore
\& Frith, 2005; Wilson \& Knoblich, 2005; Wolpert, Doya, $\&$ Kawato, 2003). Future neuroimaging studies employing paradigms like the one used here are needed to test this prediction. If one assumes that perceptual expertise and motor simulation work in concert (Canal-Bruland \& Schmidt, 2008), disrupting the functioning of motor areas in the brain through transcranial magnetic stimulation may provide a means with which to explore the specific contribution of motor expertise.

Although experts' performance was well above chance, it was by no means perfect. The most likely explanation is simply that determining when one is being deceived isn't easy. Studies of deception detection during verbal communication typically report accuracy rates below $60 \%$ (Bond \& DePaulo, 2006). Our experts were surprisingly good at detecting bodily bluffs, especially because our short-duration stimuli contained only "successful" fakes that had deceived a defender. Deception detection by experts might improve further if they responded to observed actions in a natural way (e.g., by catching the ball) rather than by making keypresses (Farrow \& Abernethy, 2003).

The present study focused on basketball, because this action domain involves extensive deception through bodily movement. Nonetheless, we expect the present results to generalize to other bodily experiences. Indeed, expertise can be regarded as a test case in that it allows one to investigate how the perception of others' actions changes as a function of the observer's repertoire (Knoblich, 2008). Basketball is also just one of many domains that lend themselves to the investigation of how mental states are inferred from perceived actions. Although little is known about how the mechanisms of mental state attribution and action perception work together (Southgate, Senju, \& Csibra, 2007), studies of how we detect bluffing bodies provide one step in that direction. If one assumes that one is best at detecting deception in actions that one performs regularly, an interesting prediction for future studies is that shoplifters should make the best store detectives.

\section{AUTHOR NOTE}

We thank John Zisa for his help in stimulus generation and data collection and Cass Foursha for starring as the basketball player in these stimuli. We thank Catherine Reed, Gabriel Diaz, and two anonymous reviewers for their helpful comments on an earlier version of the manuscript. This research was supported by NEI Grant EY12300 and NSF Grant EXP-SA 0730985. Address correspondence to N. Sebanz, Centre for Cognition, Donders Institute for Brain, Cognition, and Behaviour, Radboud University Nijmegen, P.O. Box 9104, 6500 HE Nijmegen, The Netherlands (e-mail: n.sebanz@donders.ru.nl).

\section{REFERENCES}

Abernethy, B. (1989). Expert-novice differences in perception: How expert does the expert have to be? Canadian Journal of Sport Sciences, 14, 27-30.

Abernethy, B., \& ZAWI, K. (2007). Pickup of essential kinematics underpins expert perception of movement patterns. Journal of Motor Behavior, 39, 353-367.

Behrmann, M., \& Ewell, C. (2003). Expertise in tactile pattern recognition. Psychological Science, 14, 480-486.

Blakemore, S.-J., \& Frith, C. (2005). The role of motor contagion in the prediction of action. Neuropsychologia, 43, 260-267.

Bond, C. F., JR., \& DePaulo, B. M. (2006). Accuracy of deception judgments. Personality \& Social Psychology Review, 10, 214-234. 
Bosbach, S., Cole, J., Prinz, W., \& Knoblich, G. (2005). Inferring another's expectation from action: The role of peripheral sensation. Nature Neuroscience, 8, 1295-1297.

Buccino, G., Vogt, S., Ritzl, A., Fink, G. R., Zilles, K., Freund, H.-J., \& RizzolatTi, G. (2004). Neural circuits underlying imitation learning of hand actions: An event-related fMRI study. Neuron, 42, 323-334.

Calvo-Merino, B., Glaser, D. E., Grèzes, J., Passingham, R. E., \& HAGGARD, P. (2005). Action observation and acquired motor skills: An fMRI study with expert dancers. Cerebral Cortex, 15, 1243-1249.

Calvo-Merino, B., Grèzes, J., Glaser, D. E., Passingham, R. E., \& HaGgard, P. (2006). Seeing or doing? Influence of visual and motor familiarity in action observation. Current Biology, 16, 1905-1910.

Canal-Bruland, R., \& Schmidt, M. (2008). The role of motor and perceptual expertise in the prediction of deceptive movements. Manuscript submitted for publication.

Chouchourelou, A., Matsuka, T., Harber, K., \& Shiffrar, M. (2006). The visual analysis of emotional action. Social Neuroscience, 1, 63-74.

Cross, E. S., Hamilton, A. F. De C., \& Grafton, S. T. (2006). Building a motor simulation de novo: Observation of dance by dancers. Neurolmage, 31, 1257-1267.

Cutting, J. E., \& Koslowski, L. T. (1977). Recognizing friends by their walk: Gait perception without familiarity cues. Bulletin of the Psychonomic Society, 9, 353-356.

Dittrich, W. H., Troscianko, T., Lea, S. E. G., \& Morgan, D. (1996). Perception of emotion from dynamic point-light displays represented in dance. Perception, 25, 727-738.

Ekman, P., \& Friesen, W. V. (1969). Nonverbal leakage and clues to deception. Psychiatry: Journal for the Study of Interpersonal Processes, 32, 88-106.

FARrow, D., \& Abernethy, B. (2003). Do expertise and the degree of perception-action coupling affect natural anticipatory performance? Perception, 32, 1127-1139.

FrITH, C. D., \& FrITH, U. (2006). How we predict what other people are going to do. Brain Research, 1079, 36-46.

Grèzes, J., Frith, C. D., \& PAssingham, R. E. (2004a). Brain mechanisms for inferring deceit in the actions of others. Journal of Neuroscience, 24, 5500-5505.

Grèzes, J., Frith, C. D., \& PAssingham, R. E. (2004b). Inferring false beliefs from the actions of oneself and others: An fMRI study. NeuroImage, 21, 744-750.

Heberlein, A. S., Adolphs, R., Tranel, D., \& Damasio, H. (2004). Cortical regions for judgments of emotions and personality traits from pointlight walkers. Journal of Cognitive Neuroscience, 16, 1143-1158.

JaCKSON, R. C., Warren, S., \& Abernethy, B. (2006). Anticipation skill and susceptibility to deceptive movement. Acta Psychologica, 123, 355-371.
Jacobs, A., Pinto, J., \& Shiffrar, M. (2004). Experience, context, and the visual perception of human movement. Journal of Experimental Psychology: Human Perception \& Performance, 30, 822-835.

Johansson, G. (1973). Visual perception of biological motion and a model for its analysis. Perception \& Psychophysics, 14, 201-211.

KNOBLICH, G. (2008). Bodily and motor contributions to action perception. In R. Klatzky, B. MacWhinney, \& M. Behrmann (Eds.), Embodiment, ego-space, and action (pp. 45-78). New York: Psychology Press.

KNOBLICH, G., \& FlaCH, R. (2001). Predicting the effects of actions: Interactions of perception and action. Psychological Science, 12, 467-472.

Loula, F., Prasad, S., Harber, K., \& Shiffrar, M. (2005). Recognizing people from their movement. Journal of Experimental Psychology: Human Perception \& Performance, 31, 210-220.

Müller, S., Abernethy, B., \& Farrow, D. (2006). How do worldclass cricket batsmen anticipate a bowler's intention? Quarterly Journal of Experimental Psychology, 59, 2162-2186.

Rizzolatti, G., \& Craighero, L. (2004). The mirror-neuron system. Annual Review of Neuroscience, 27, 169-192.

Runeson, S., \& FRYKHOLM, G. (1983). Kinematic specification of dynamics as an informational basis for person-and-action perception: Expectation, gender recognition, and deceptive intention. Journal of Experimental Psychology: General, 112, 585-615.

Southgate, V., Senju, A., \& Csibra, G. (2007). Action anticipation through attribution of false belief by 2 -year-olds. Psychological Science, 18, 587-592.

Troje, N. F., \& BüLthoff, H. H. (1996). Face recognition under varying poses: The role of texture and shape. Vision Research, 36, 1761-1771.

Troje, N. F., Westhoff, C., \& Lavrov, M. (2005). Person identification from biological motion: Effects of structural and kinematic cues. Perception \& Psychophysics, 67, 667-675.

VRIJ, A. (2004). Why professionals fail to catch liars and how they can improve. Legal \& Criminological Psychology, 9, 159-181.

VRIJ, A. (2006). Nonverbal communication and deception. In V. Manusov \& M. L. Patterson (Eds.), The Sage handbook of nonverbal communication (pp. 341-359). Thousand Oaks, CA: Sage.

Wilson, M., \& KNOBLich, G. (2005). The case for motor involvement in perceiving conspecifics. Psychological Bulletin, 131, 460-473.

Wolpert, D. M., Doya, K., \& Kawato, M. (2003). A unifying computational framework for motor control and social interaction. Philosophical Transactions of the Royal Society B, 358, 593-602.

(Manuscript received April 30, 2008; revision accepted for publication August 11, 2008.) 